\title{
Macroscopic loop formation in circular DNA denaturation
}

\author{
Alkan Kabakçıŏlu, ${ }^{1}$ Amir Bar, ${ }^{2,3}$ and David Mukamel $^{2}$ \\ ${ }^{1}$ Department of Physics, Koç University, Sartyer 34450 Istanbul, Turkey \\ ${ }^{2}$ Department of Physics of Complex Systems, The Weizmann Institute of Science, Rehovot 76100, Israel \\ ${ }^{3}$ Department of Computer Science and Applied Mathematics, The Weizmann Institute of Science, Rehovot 76100, Israel
}

(Received 1 April 2012; published 29 May 2012)

\begin{abstract}
The statistical mechanics of DNA denaturation under fixed linking number is qualitatively different from that of unconstrained DNA. Quantitatively different melting scenarios are reached from two alternative assumptions, namely, that the denatured loops are formed at the expense of (i) overtwist or (ii) supercoils. Recent work has shown that the supercoiling mechanism results in a picture similar to Bose-Einstein condensation where a macroscopic loop appears at $T_{c}$ and grows steadily with temperature, while the nature of the denatured phase for the overtwisting case has not been studied. By extending an earlier result, we show here that a macroscopic loop appears in the overtwisting scenario as well. We calculate its size as a function of temperature and show that the fraction of the total sum of microscopic loops decreases above $T_{c}$, with a cusp at the critical point.
\end{abstract}

DOI: 10.1103/PhysRevE.85.051919

PACS number(s): 87.15.Zg, 36.20.Ey

The melting or denaturation of DNA refers to the separation of the two complementary DNA strands, a process that can be induced either thermally [1] or mechanically [2-4]. The associated phase transition is well understood by means of theoretical models, see, e.g., the models by Poland and Scheraga [5], Peyrard and Bishop [6], and their various extensions [7-9]. Thermal melting of DNA forms the basis of the polymerase chain reaction (PCR) technique, while statistical and dynamical properties of denatured loops may turn out to be relevant to understanding DNA-protein interactions $[10,11]$ and gene expression initiation [12,13].

Interestingly, it is a common practice to use plasmid (circular) DNA molecules during a PCR since most bacteria come with circular DNA as a means of protection against degradation. The resulting entanglement of the two strands due to the natural twist of the DNA molecule imposes an obvious obstacle for the denaturation process (as well as replication, protein synthesis, etc.), which is overcome in nature by means of special DNA manipulating proteins [14]. Nevertheless, the thermal behavior of a circular DNA chain in the absence of such helper proteins proves to be a nontrivial problem and has been addressed recently [15-17].

The circular geometry entails the presence of a new topological invariant in the system: the number of times two chains of the DNA wind around each other, namely, the linking number (LN). The thermodynamics of the system should therefore be investigated within the corresponding restricted phase space. This framework is also relevant to singlemolecule experiments on DNA in which the chain ends are rotationally constrained [18]. We discuss here the implications of LN conservation on thermal melting characteristics within the framework of the Poland-Scheraga (PS) model.

A consequence of fixing the linking number is that the denatured loops form at the expense of (right-handed) torsional stress on surrounding DNA duplex segments. As with any elastic ribbon with a finite bending and twist modulus, dsDNA responds to torsion by supercoiling (bending the backbone as in coiling telephone cords) and/or by overtwisting (modifying the stacking angle). The thermodynamics of a fixed-LN DNA chain whose bound segments are unbendable but have finite twist rigidity was investigated by Rudnick and Bruinsma [15]. An alternative extension of the PS model considering the possibility of supercoil formation, but not overtwisting, has also been discussed recently [17,19]. It has been shown that the transition is a kind of Bose-Einstein condensation (BEC) where the macroscopic loop formed above the melting temperature plays the role of the condensate.

We show below that a BEC-like transition takes place in the overtwisting scenario too, which is the main contribution of this paper. We next compare this phenomenon with a similar observation we made earlier for the supercoiling response and with the denaturation of DNA with free ends. We conclude that the birth of a nontrivial macroscopic loop at the melting point is the defining characteristic of the thermal denaturation of DNA under fixed linking number, irrespective of how the molecule responds to torsional stress.

Reference [20] gives a detailed account of the melting transition in the PS model. The partition function of the model can be expressed in closed form and its singular behavior, with proper treatment of the loop entropy, yields a first-order melting transition. A similar analysis is given in Refs. [17,19] for the case of nonzero supercoil density under the constraint that the total length of the denatured loops is proportional to that of supercoils (mimicking LN conservation). This system, unlike the PS model, displays a continuous melting transition, accompanied by a loop condensate that appears at $T_{c}$ and grows gradually with temperature.

Overtwisting, i.e., increasing the stacking angle between the successive base pairs, is the alternative to supercoiling, by which a partially denatured circular DNA chain can accommodate the resulting torsional stress on duplex regions. An extension of the PS model with overtwist has been investigated earlier in Ref. [15], which we extend below. In particular, we show here that the melting transition in this scenario too is accompanied by the formation a macroscopic loop.

To this end, following Ref. [15], let us consider an arbitrary configuration of a DNA chain of a total length $L$ composed of denatured segments with total length $L_{l}$ and bound segments with $L_{b}=L-L_{l}$. We assume that the linking number 
expelled by the loops is uniformly distributed along the chain and results in a uniform increase $\delta \theta \propto L_{l} / L_{b}$ in the stacking angle measured between the two ends of a unit DNA segment. Associated overtwist energy per unit length is assumed to be harmonic and the total internal energy can be written as

$$
\mathcal{H}=\kappa \frac{L_{l}^{2}}{L_{b}}+\epsilon_{b} L_{b},
$$

where $\kappa>0$ is a measure of overtwist stiffness in units of energy per length and $\epsilon_{b}<0$ is the binding energy per unit length. The canonical partition function for the DNA chain can then be expressed as [15]

$$
Z^{\kappa}\left(L_{b}, L_{l}\right)=\oint \frac{d z_{b} d z_{l}}{(2 \pi i)^{2}} \frac{Q^{\kappa=0}\left(z_{b}, z_{l}\right)}{z_{b}^{L_{b}+1} z_{l}^{L_{l}+1}} e^{-\beta \kappa\left(L_{l}^{2} / L_{b}\right)},
$$

where the grand sum $Q^{\kappa=0}=\left[1 / \omega z_{b}-1-A \Phi_{c}\left(s z_{l}\right)\right]^{-1}$ follows from the usual PS model with the Boltzmann weight $\omega$ for a unit bound segment and fugacities $\left\{z_{b}, z_{l}\right\}$ per unit length of bound and denatured DNA, respectively. The cooperativity parameter $A, s$, and the loop exponent $c$ are constants associated, in given order, with the loop initiation cost, the leading contribution to the loop entropy, and the subleading universal correction to the loop entropy [20] in the Boltzmann weight $A s^{l} / l^{c}$ of a loop of length $l$. The contour integral in Eq. (2) has a simple pole for $z_{b}$, which by using the Cauchy formula yields

$$
\begin{aligned}
Z^{\kappa}\left(L_{b}, L_{l}\right)= & \frac{1}{2 \pi i} \oint \frac{d z_{l}}{z_{l}^{L_{l}+1}} e^{-\beta \kappa\left(L_{l}^{2} / L_{b}\right)} \omega^{L_{b}} \\
& \times\left[1+A \Phi_{c}\left(s z_{l}\right)\right]^{L_{b}-1} \equiv \oint d z_{l} e^{-L F\left(z_{l}, m_{l}\right)},
\end{aligned}
$$

with

$$
\begin{aligned}
F\left(z_{l}, m_{l}\right)= & -\left(1-m_{l}\right) \ln \left\{\omega\left[1+A \Phi_{c}\left(s z_{l}\right)\right]\right\} \\
& +m_{l} \ln z_{l}+\beta \kappa \frac{m_{l}^{2}}{1-m_{l}}+O\left(L^{-1}\right),
\end{aligned}
$$

where $m_{l, b}=L_{l, b} / L$ and $m_{l}+m_{b}=1$. In the thermodynamic limit the partition function can be evaluated using the saddle-point condition $\partial F=0$. Therefore, $F\left(z_{l}, m_{l}\right)$ serves as a free energy functional for the DNA chain. Minimization yields a continuous phase transition for $c>2$ governed by the singularity of the polylogarithm function at $s z=1$ [15]. It is straightforward to show that the critical temperature $T_{c}$ shifts linearly with the ratio of the overtwist penalty $\kappa$ and the binding energy $\epsilon_{b}=-k_{B} T \ln \omega$ :

$$
T_{c}=T_{c}^{\mathrm{PS}}\left[1-\frac{\kappa}{\epsilon_{b}}\left(\frac{1}{\left(1-m_{l}^{c}\right)^{2}}-1\right)\right] .
$$

Here $T_{c}^{\mathrm{PS}}$ is the critical temperature of the original PS model and $m_{l}^{c}$ is the critical loop fraction, which we find to be independent of the twist stiffness (therefore equal to the corresponding value in the PS model):

$$
m_{l}^{c}=\frac{A \zeta_{c}}{1+A\left(\zeta_{c}+\zeta_{c-1}\right)} .
$$

Here $\zeta_{c}=\Phi_{c}(1)$ is the Riemann zeta function.
Our goal is to investigate the existence of a macroscopic loop for $T>T_{c}$ in the above picture. Let us assume that such a loop exists with size $L_{0} \equiv m_{0} L$ and calculate $m_{0}$. As for a Bose gas below the condensation temperature, the estimated amount of denatured DNA, when calculated as a sum over microscopic loops, is now short of the actual value by $L_{0}$. Therefore we set $L_{l}=L_{l}^{\text {micro }}+L_{0}$ and substitute in Eq. (2)

$$
Z^{\kappa}\left(L_{l}, L_{b}\right) \rightarrow Z^{\kappa}\left(L_{l}-L_{0}, L_{b}\right) \frac{s^{L_{0}}}{L_{0}^{c}},
$$

which, following the same steps, now yields an additive macroscopic loop correction to Eq. (3):

$$
F\left(z_{l}, m_{l}, m_{0}\right)=F\left(z_{l}, m_{l}\right)-m_{0} \ln \left(s z_{l}\right) .
$$

Note that $m_{l}$ is the total loop density, including microscopic and macroscopic contributions.

For $T<T_{c}\left(z_{l}<1 / s\right)$, the free energy is minimized with respect to $m_{0}$ at the extremal value $m_{0}=0$. Therefore no macroscopic loop exists below $T_{c}$. The total fraction of denatured bases (all due to microscopic loops) can be calculated by setting $\partial_{m_{l}} F=\partial_{z_{l}} F=0$. It increases with temperature slower than in the PS model due to the additional (overtwist) energy penalty of denaturation. The loop-size distribution essentially decays exponentially with a power-law correction as in the PS model.

In the high-temperature phase where the loop fugacity is fixed at its upper bound $z_{l}=1 / s$, the scenario above is no longer valid. The minimum of the free energy in Eq. (3) is now given by $\partial F\left(z_{l}, m_{l}, m_{0}\right)=0$, i.e.,

$$
\begin{aligned}
& 0=\frac{\partial F}{\partial m_{0}} \Rightarrow z_{l}=1 / s, \\
& 0=\frac{\partial F}{\partial m_{l}} \Rightarrow \frac{\omega}{s}\left(1+A \zeta_{c}\right)=e^{-\beta \kappa\left[-1+1 /\left(1-m_{l}\right)^{2}\right]}, \\
& 0=\frac{\partial F}{\partial z_{l}} \Rightarrow m_{l}=\frac{m_{0}+R}{1+R},
\end{aligned}
$$

where $R \equiv \frac{A \zeta_{c-1}}{1+A \zeta_{c}}$ is a temperature-independent constant. These equations yield a unique solution with $m_{0} \neq 0$ (the extremal value $m_{0}=0$ does not yield a minimum). Therefore, a finite fraction of the base pairs is located in a macroscopic loop. A consequence of Eq. (7) is that the probability distribution function for the microscopic loop sizes $p(l) \sim l^{-c}$ is now scale invariant. This observation is in contrast with the PS model, since no microscopic loop survives in the PS model for $T>T_{c}$.

An analytical expression for the mass fraction in microloops and the macroloop can be obtained from Eqs. (8) and (9). Solving for $m_{0}$, then substituting $\ln \omega=-\epsilon_{b} / k_{B} T$, and using Eq. (4) one finds that the macroscopic loop fraction has the exact form

$$
m_{0}=1-\frac{1}{\sqrt{1+B\left(T-T_{c}\right)}},
$$

where $B=-\frac{\epsilon_{b}}{\kappa T_{c}}(1+R)^{-2}$. The macroscopic loop fraction $m_{0}$ grows linearly with temperature in the vicinity of $T_{c}$ and 


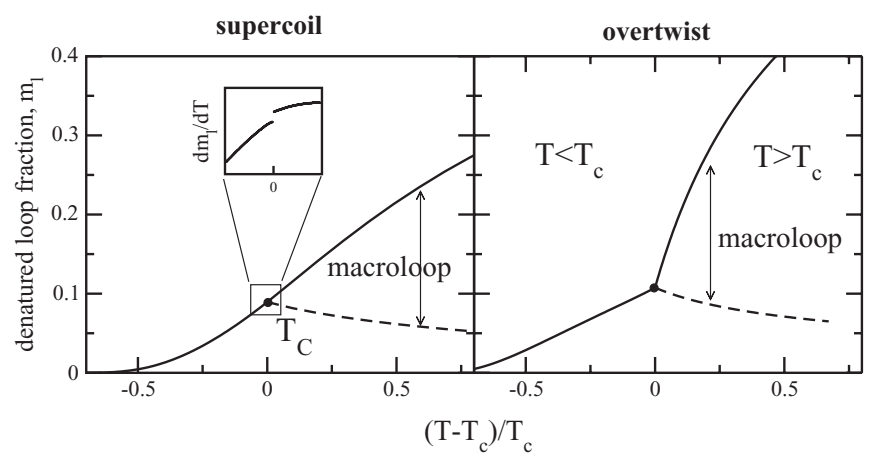

FIG. 1. Fraction of the denatured DNA (solid line) and the contribution from the microscopic loops (dashed line) for the supercoiling (left) and overtwisting (right) scenarios, as a function of temperature. The parameters $s=5, \epsilon_{b}=3, c=3.5$, and $A=0.1$ are the same for both figures, while the stiffness parameters for overtwisting $(\kappa=1.0)$ and supercoiling (see Ref. [19]) are comparable.

approaches unity only in the limit $T \rightarrow \infty$. The resulting transition is continuous since setting $m_{0}=0\left(T \rightarrow T_{c}^{+}\right)$ recovers the critical value of $m_{l}$ in Eq. (5) found from the lowtemperature limit $T \rightarrow T_{c}^{-}$. The total fraction of microscopic loops decreases with temperature while their distribution remains a power law. In Fig. 1 we present a comparison of the overtwist and the supercoil dominated scenarios in terms of macroscopic and microscopic loop fractions for a generic set of parameters for which both display a second-order melting transition. Note that the discontinuity in $d m_{l} / d T$ at $T=T_{c}$ (inset) in the supercoiling model is hardly visible. However, the cusp in the microscopic loop fraction is clear and exists even for $c<3$, where the discontinuity at $T_{c}$ shifts to higher derivatives of the free energy (see Ref. [19] for more on the PS model with supercoiling). The cusp in $m_{l}$ is more prominant in the overtwist picture with a comparable set of parameters.

The presence of the macroloop above $T_{c}$ does not depend on the precise value of $c$ (as long as there is a melting transition), while its size relative to the total amount of denatured DNA $m_{0} / m_{l}$ does (Fig. 2). For $c \leqslant 2$ the macroscopic loop vanishes together with the transition itself. Otherwise, the relative size

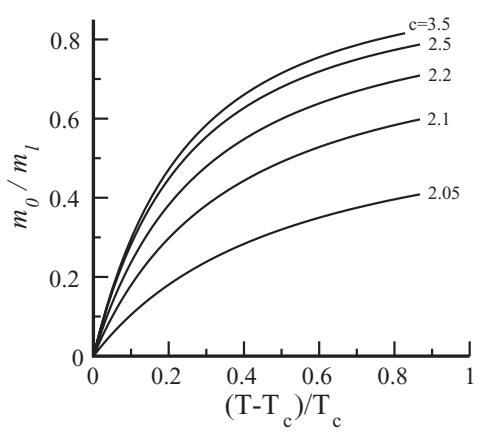

FIG. 2. Weight of the macroloop among all the denatured bases shown as a function of temperature for various values of $c$. The top curve for $c=3.5$ is indistinguishable from those obtained for higher values of $c$.

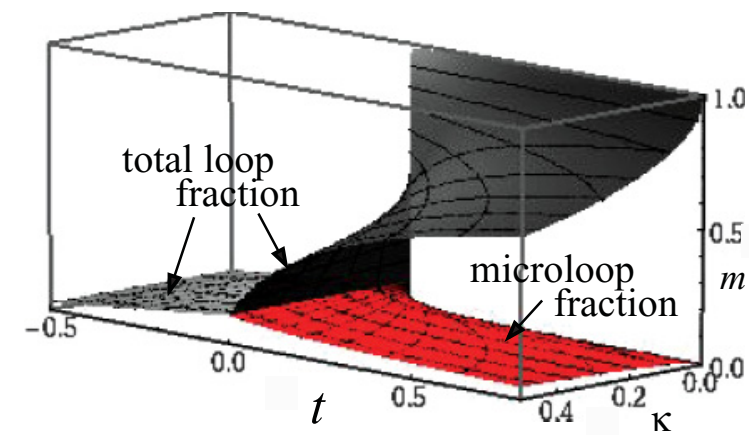

FIG. 3. (Color online) Loop fraction $m$ of the DNA as a function of the reduced temperature $t=\left(T-T_{c}\right) / T_{c}$ and the overtwist penalty $\kappa$. The surface labeled "microloop fraction" (red online) shows the contribution of the microscopic loops to the total denatured DNA. The size of the macroscopic loop is the vertical distance between the top (gray) and the bottom (red) surfaces for $t>0$. The back panel of the bounding box corresponds to the PS model. Note that the reduced temperature $t$ does not correspond to a single temperature value since $T_{c}$ is a function of $\kappa$.

of the macroloop grows faster with temperature as $c$ gets larger. This trend as a function of $c$ converges to a limiting curve that is indistinguishable from that obtained for $c=3.5$ and shown in Fig. 2.

Generalization of our results to $\sigma \equiv\left(L_{s}-L_{l}\right) / L \neq 0$ is straightforward after substituting $\beta \kappa\left(m_{l}+\sigma\right)^{2} /\left(1-m_{l}\right)$ for the twist penalty in Eq. (3). The melting picture for nonzero $\sigma$ remains qualitatively unaltered except when $\sigma \leqslant-m_{l}^{c}$, where the critical point itself disappears and a macroscopic loop exists at all temperatures.

Finally, noting that the limit $\kappa \rightarrow 0$ recovers the first-order transition, it is interesting to see how the sharp denaturation in the PS model smooths out with the introduction of a twist penalty. This crossover is shown in Fig. 3, where the total loop fraction given in Fig. 1 is extended into the $\kappa$ dimension. The region between the two sheets that join at the critical line $t=0$ is the macroscopic loop fraction, which smoothly approaches unity as $\kappa \rightarrow 0$.

To summarize, we reconsidered the melting thermodynamics of a DNA chain with fixed linking number. We assumed that the denatured loops appear by transferring the LN to duplex regions through overtwisting. We showed that, despite the different melting scenarios observed in supercoiling and overtwisting pictures, a feature common to both is the appearance of a macroscopic loop that grows monotonically with temperature. While the total fraction of denatured pairs increases with temperature, the DNA mass in the microscopic loops decreases above $T_{c}$. This condensation phenomenon is analogous to BEC, except that it takes place at high temperature. Whether it is dynamically accessible is an interesting question since merging microscopic loops toward a macroloop entails diffusing denaturation bubbles across torsionally strained duplex regions.

This work has been supported by the Scientific and Technological Research Council of Turkey through Grant No. TBAG-110T618 and by the Israeli Science Foundation. 
[1] R. M. Wartell and A. S. Benight, Phys. Rep. 126, 67 (1985).

[2] I. Rouzina and V. A. Bloomfield, Biophys. J. 80, 882 (2001).

[3] S. Cocco, R. Monasson, and J. F. Marko, Proc. Natl. Acad. Sci. USA 98, 8608 (2001).

[4] D. Marenduzzo, A. Trovato, and A. Maritan, Phys. Rev. E 64, 031901 (2001).

[5] D. Poland and H. A. Scheraga, J. Chem. Phys. 45, 1456 (1966).

[6] M. Peyrard and A. R. Bishop, Phys. Rev. Lett. 62, 2755 (1989).

[7] A. Hanke, M. G. Ochoa, and R. Metzler, Phys. Rev. Lett. 100, 018106 (2008).

[8] T. Dauxois, M. Peyrard, and A. R. Bishop, Phys. Rev. E 47, 684 (1993).

[9] R. D. Blake, J. W. Bizzaro, J. D. Blake, G. R. Day, S. G. Delcourt, J. Knowles, K. A. Marx, and J. SantaLucia Jr., Bioinformatics 15, 370 (1999).

[10] G. Altan-Bonnet, A. Libchaber, and O. Krichevsky, Phys. Rev. Lett. 90, 138101 (2003).

[11] R. A. Forties, R. Bundschuh, and M. G. Poirier, Nucleic Acids Res. 37, 4580 (2009).
[12] G. Kalosakas, K. Ø. Rasmussen, A. R. Bishop, C. H. Choi, and A. Usheva, Europhys. Lett. 68, 127 (2004).

[13] T. S. Van Erp, S. Cuesta-Lopez, J. G. Hagmann, and M. Peyrard, Phys. Rev. Lett. 95, 218104 (2005).

[14] B. Alberts, D. Bray, A. Johnson, J. Lewis, M. Raff, K. Roberts, P. Walter, and A. M. Campbell, Essential Cell Biology (Garland Science, New York, 2004).

[15] J. Rudnick and R. Bruinsma, Phys. Rev. E 65, 030902(R) (2002).

[16] T. Garel, H. Orland, and E. Yeramian, arXiv:q-bio/0407036.

[17] A. Kabakçığlu, E. Orlandini, and D. Mukamel. Phys Rev E 80, 010903(R) (2009).

[18] Z. Bryant, M. D. Stone, J. Gore, S. B. Smith, N. R. Cozzarelli, and C. Bustamante, Nature (London) 424, 338 (2003).

[19] A. Bar, A. Kabakcioglu, and D. Mukamel, Phys. Rev. E 84, 041935 (2011).

[20] Y. Kafri, D. Mukamel, and L. Peliti, Phys. Rev. Lett. 85, 4988 (2000). 